\title{
MODELAGEM MATEMÁTICA DE SENSORES PIEZORESISTIVOS BASEADOS EM FILMES FINOS SEMICONDUTORES
}

\author{
ALBERTO MOI ${ }^{1}$, RODRIGO MOREIRA ${ }^{1}$, LUIZ ANTONIO RASIA $^{2}$.
}

1. DCEEng, Departamento de Ciências Exatas e Engenharias, Unijui. Rua São Francisco, 501 Caixa Postal 560, 98700, Ijuí, RS,BRASIL

E-mails: betomoiilegmail.com, jpceditors@msn.com

\section{DCEEng, Departamento de Ciências Exatas e Engenharias, Unijui. Rua São Francisco, 501 Caixa Postal 560, 98700, Ijuí, RS,BRASIL \\ E-mails: rasia@unijui.edu.br.}

\begin{abstract}
This paper presents the theoretical studies on the characterization of the piezoresistive effect in semiconductor thin films by developing mathematical models and computer simulations, aiming to develop an Integrated Testing Environment for simulation and optimization parameters of electrical, thermal and mechanical processes used in manufacture of integrated piezoresistive sensors.
\end{abstract}

Keywords—Mathematical modeling, sensor elements, piezoresistors, semiconductor films.

Resumo-Este trabalho apresenta os estudos teóricos sobre a caracterização do efeito piezoresistivo em filmes finos semicondutores através do desenvolvimento de modelos matemáticos e simulações computacionais, visando desenvolver um Ambiente Integrado de Testes para simulação e otimização de parâmetros elétricos, térmicos e mecânicos usados nos processos de fabricação de sensores piezoresistivos integrados.

Palavras-chave—Modelagem Matemática, elementos sensores, piezoresistores, filmes semicondutores.

\section{Introdução}

O efeito da piezoresistividade consiste na alteração da resistência elétrica de um material quando sobre ele é aplicada uma determinada tensão mecânica,

A descoberta deste fenômeno deve-se ao matemático, físico e engenheiro britânico Lord Kelvin, que em 1856 verificou que a resistência de fios de cobre e ferro aumentava quando sobre eles era aplicada uma determinada tensão mecânica (Rasia, 1997).

Atualmente, o efeito piezoresistivo vem sendo estudado em materiais semicondutores e está bem estabelecido para o silício, em especial, nos dispositivos fabricados com a tecnologia de filmes finos.

Para o desenvolvimento de elementos sensores é necessário compreender como se comportam as propriedades físicas do silício, no qual a rede cristalina é considerada simétrica. Nestes materiais os mecanismos mais importantes e que limitam a mobilidade, $\mu$, de portadores livres de cargas elétricas são o espalhamento pelas impurezas ionizadas e o espalhamento pelas vibrações da rede, (Rasia, 1997). Nestes fenômenos os átomos vibram em torno de uma posição de equilíbrio, cuja vibração provoca uma variação de energia dos extremos das bandas de condução e valência em função da constante da rede e se devem a modulação da constante devido às vibrações da própria estrutura.
Em materiais semicondutores existem diferentes tipos de estruturas de banda com diferentes formas de superfícies de energia no espaço de onda, $\mathrm{K}$. Estas superfícies se modificam e promovem uma mudança da resistividade, $\rho$, do material. Para o silício o modelo de superfície de energia que parece ocorrer, para a banda de condução elétrica, é a ilustrada na figura 1 e figura 2, cujo processo físico é denominado intervales (intervalley), (Vlack, 1973).

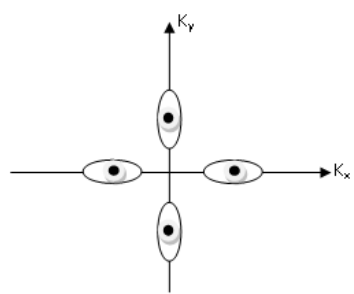

Figura 1. Ilustração de superfície de energia para o silício não estressado no momento de espaço K.

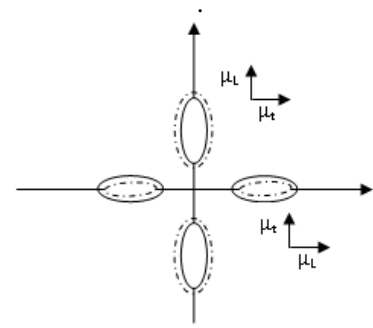

Figura 2. Ilustração de superfície de energia para o silício estressado no momento de espaço $\mathrm{K}$. 
Nos últimos anos, outros tipos de materiais, tais como, DLC, ITO, SiC entre outros, vêm sendo estudados e usados em ambientes corrosivos e de alta temperatura. Estes materiais são empregados onde o silício não pode ser usado.

A resposta da alteração na resistividade em materiais semicondutores pode ser expressa em termos de um tensor com três componentes independentes denominados de coeficientes piezoresistivos, $\pi_{11}, \pi_{12}$ e $\pi_{44}$. Os coeficientes piezoresistivos estão relacionados com a alteração na resistividade devido ao esforço mecânico ou estresse mecânico e podem ser convertidos para deformações mecânicas ou "strain", através do uso do modulo de Young (French, 1989, Dally, 1978), de acordo com a equação:

$$
E=\frac{T}{\epsilon}
$$

onde, $E$ é o modulo de elasticidade, $T$ é tensão mecânica aplicada, ambos medidos em pascal e $\varepsilon$ é a deformação elástica longitudinal do corpo de prova (adimensional).

Monocristais de silício são elasticamente anisotrópicos (Ciureanu, 1992) e devem ser descritos através da equação (2).

$$
\frac{\Delta \rho i j}{\rho}=\sum_{k . l} \pi_{I J K L} T_{K L}
$$

Onde, $\frac{\Delta \rho i j}{\rho}$ é o efeito piezoresistivo direto.

É possível fazer adequações nas equações (1) e (2) para aplicá-las em um material policristalino ou mesmo amorfo (Rasia, 1997).

Introduz-se uma notação equivalente matricial de uso generalizado na literatura para facilitar a reduzir o número de elementos independentes do tensor, de modo que a equação (2) fica:

$$
\frac{\Delta \rho i}{\rho}=\sum_{j=1}^{6} \pi_{i j} T j
$$

Assim, para $\mathrm{i}=1, \ldots 6$ o tensor de coeficientes piezoresistivos (Ciureanu, 1992, Bau, vol.7) é dado pela matriz:

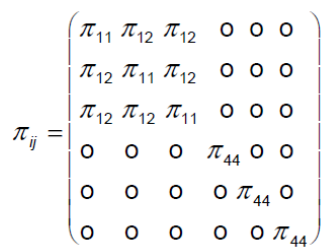

Os coeficientes piezoresistivos, $\pi_{\mathrm{s}}$ para material tipo $\mathrm{P}$ e tipo $\mathrm{N}$, estão relacionados com os níveis de concentração de impurezas dopantes, orientação cristalográfica do material, temperatura e, consequentemente, com o tipo de condutividade, (Akbar, 1991), figura 3 e figura 4.

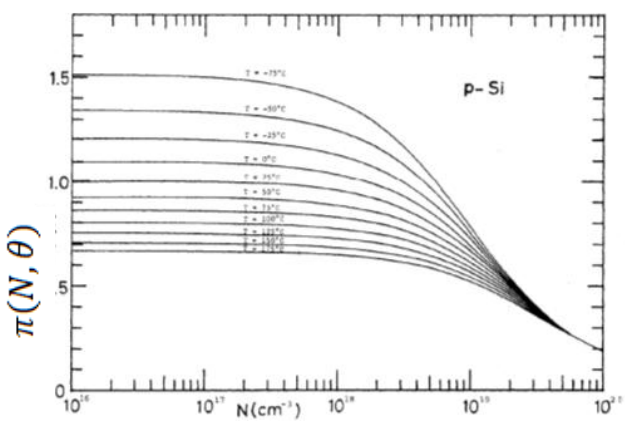

Figura 3. Coeficiente de Piezoresistência para material tipo P

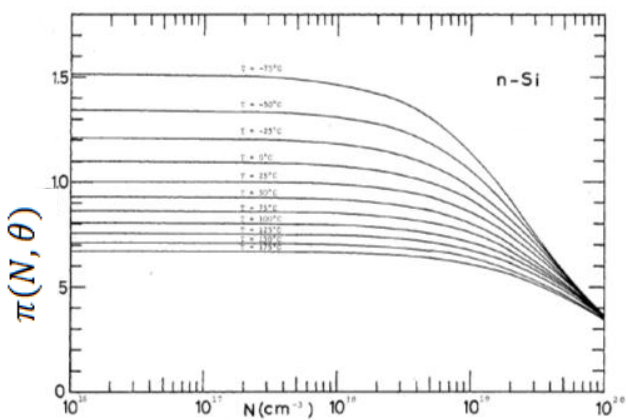

Figura 4. Coeficiente de Piezoresistência para material tipo N

Estes parâmetros são importantes para os processos tecnológicos de fabricação dos dispositivos sensores e atuadores uma vez que estão relacionados com a sensibilidade dos dispositivos (Rasia, 1997, Maisel, 1983, Gniazdowiski, 1998).

\subsection{Propriedades dos Materiais Semicondutores}

O desenvolvimento de estruturas ou sistemas micro-eletro-mecânicos, MEMS, dependem criticamente da disponibilidade de materiais semicondutores com propriedades apropriadas para as diversas aplicações destes dispositivos. Para a fabricação de sensores e atuadores é necessário o conhecimento das inter-relações ou efeitos cruzados existentes entre as diversas grandezas e parâmetros físicos dos materiais envolvendo efeitos elétricos, térmicos, mecânicos e ópticos. Estas propriedades podem ser ajustadas durante os processos de difusão, deposição ou implantação iônica (Rasia, 1997).

\subsection{Materiais semicondutores}

Os semicondutores são do grupo de materiais elétricos possuindo condutividade elétrica, $\sigma$, intermediária entre metais e isolantes. A magnitude desta propriedade pode ser variada em quantidades controladas através da mudança do nível de concentração de impurezas, temperatura e excitação óptica, durante os processos de fabricação.

A concentração de impurezas, $\mathrm{N}$, é usada para alterar a condutividade dos semicondutores de modo que os mesmos podem se tornar materiais tipo $\mathrm{N}$ ou 
tipo $\mathrm{P}$, dependendo do dopante como sugere (Kanda, 1982).

O silício é usado para a fabricação de diferentes dispositivos semicondutores, sensores e atuadores devido ao domínio dos processos de fabricação e as suas excelentes propriedades físicas.

Neste trabalho, serão estudados elementos sensores com substrato de silício e deposição de diferentes filmes finos semicondutores, de acordo com o modelo físico mostrado na figura 5, de acordo com (Maisel, 1983, Gniazdowiski, 1998)

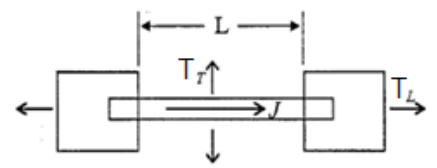

Figura 5. Modelo físico de um piezoresistor

Num elemento sensor, como o mostrado na figura 3, a mudança da resistividade é descrita pela mudança relativa da resistência elétrica do resistor quando submetido a um estresse mecânico externo aplicado. Dessa forma, o modelo matemático que descreve esta variação da resistência elétrica pode ser reescrito e relacionado com os componentes longitudinais e transversais do estresse mecânico e com os respectivos coeficientes piezoresistivos do material do qual é feito o elemento sensor, de acordo com a equação:

$$
\frac{\Delta R}{R}=\left(\frac{\Delta \rho}{\rho}\right)=\pi_{l} T_{l}+\pi_{t} T_{t}
$$

Sendo que $\pi_{l}$ e $\pi_{t}$ são os coeficientes piezoresistivos longitudinais e transversais e $T_{l}$ e $T_{t}$ são os esforços mecânicos longitudinais e transversais ao eixo de aplicação da força sobre o cristal semicondutor.

Para investigar estas úteis propriedades dos semicondutores e aperfeiçoar a funcionalidade dos dispositivos sensores e atuadores são necessários compreender a combinação atômica nestes materiais, os quais podem ser policristalinos, monocristalinos e mesmo amorfos.

\subsection{Material policristalino}

É um sólido constituído de uma infinidade de cristais denominados grãos ou cristalitos, com orientações cristalográficas e dimensões aleatórias, fortemente unidos entre si, que preenchem todo o volume do sólido (Vlack, 1973). As áreas onde estes grãos encontra-se são conhecidas como contornos de grão e são usados para processos de fabricação de diferentes tipos de sensores e atuadores devido as suas bem estabelecidas propriedades elétricas, térmicas e mecânicas.

\subsection{Material monocristalino}

É um material no qual a rede cristalina da amostra inteira é contínua e sem rupturas até suas bordas, sem contornos ou fronteiras de grãos. A ausência de defeitos associados com contornos de grãos pode dar aos monocristais propriedades únicas, particularmente, mecânicas, elétricas, térmicas e ópticas, as quais podem também ser anisotrópicas, dependendo do tipo de estrutura cristalográfica. Estas propriedades, são exploradas em aplicações tecnológicas, especialmente, em óptica e eletrônica e, neste trabalho, serão explorados para fabricação de elementos sensores (wikipedia, 2012).

\subsection{Material amorfo}

É a designação dada à estrutura que não têm ordenação espacial ou orientação cristalográfica preferêncial a longa distância, quando comparados aos sólidos regulares. Os materiais amorfos não possuem estrutura atômica definida e apresentam fácil magnetização devida ao fato de seus átomos se encontrarem arranjados de maneira aleatória, facilitando a orientação dos domínios magnéticos. Neste trabalho, estes materiais serão explorados do ponto de vista do uso das propriedades piezoresistivas no projeto de elementos sensores (Vlack, 1973).

\section{Características Físicas dos filmes finos}

Os filmes finos desempenham uma função essencial nos dispositivos e circuitos integrados, eles são usados nas conexões das regiões ativas de um dispositivo, na comunicação entre dispositivos, no acesso externo aos circuitos, para isolar camadas condutoras, como elementos estruturais dos dispositivos, para proteger as superfícies do ambiente externo, como fonte de dopante e como barreira para a dopagem. Os filmes finos podem ser caracterizados como condutores, semicondutores ou isolantes, normalmente, são crescidos termicamente ou depositados a partir da fase vapor.

A espessura, a estrutura atômica e a composição química destes filmes devem ser uniformes, com baixa densidade de defeitos e mínima contaminação por partículas para que seja mantida a funcionalidade dos dispositivos fabricados.

As geometrias diminutas dos dispositivos semicondutores resultam em circuitos com superfícies bastante rugosas. Neste caso, os filmes depositados devem ter boa aderência, baixo estresse mecânico e prover uma boa cobertura de degraus. Normalmente, as propriedades de um material na forma de filme fino diferem substancialmente das propriedades do mesmo material na sua forma maciça devido à influência da superfície. Por outro lado, as propriedades dos filmes são altamente dependentes dos processos de deposição. Estes parâmetros descritos devem ser considerados para a fabricação e processamento de elementos sensores piezoresistivos, uma vez, que os dispositivos sensores usam as mesmas técnicas de microeletrônica (Unicamp, 2013). 


\subsection{Controle da estrutura dos filmes semicondutores}

Normalmente, os filmes são formados por grãos monocristalinos dispostos em várias direções cristalográficas. O tamanho dos grãos depende das condições da deposição e dos tratamentos térmicos posteriores e os grãos com dimensões maiores, geralmente, estão associados a temperaturas maiores de processamento.

A rugosidade de um filme está relacionada com o tamanho dos grãos. A densidade de um filme pode dar informações sobre sua estrutura física e densidades menores que a da substância bruta indica que os filmes possuem porosidade (Furlan, 1997).

\section{Propriedades mecânicas}

\subsection{Coeficientes Elásticos dos Filmes}

Em muitos casos os esforços mecânicos são componentes ao longo do eixo do cristal semicondutor empregado para fabricação de elementos sensores, porém, em outros casos, trabalha-se em um sistema de coordenadas orientadas arbitrariamente, de modo que, deve ser usada a Lei de Hooke generalizada para determinar os coeficientes de deformação elásticas, $S_{i j k l}$, expressados através da equação:

$$
\varepsilon_{i j}=S_{i j k l} T_{i j}
$$

Sendo que $S_{i j k l}$, é um tensor de quarta ordem de constantes de deformações elásticas do substrato ou filme considerado. O conhecimento das constantes é fundamental para produzir diafragmas a partir de um determinado material, uma vez que, é possível determinar as relações que existem entre o esforço mecânico e a deformação mecânica e, consequentemente, o comportamento de um piezoresistor que venha a ser fabricado sobre um determinado substrato, cujos valores fundamentais ao longo dos eixos do cristal são: $s_{11}=0,764 \times 10^{-12} \mathrm{~cm}^{2} /$ dinas, $s_{12}=$ $0,214 \times 10^{-12} \mathrm{~cm}^{2} /$ dinas e $s_{44}=1,256 \times 10^{-12}$ $\mathrm{cm}^{2} /$ dinas para filmes de silício (Rasia, 1997).

$\mathrm{Na}$ forma matricial, a equação (6) pode ser escrita da seguinte forma:

$$
\left[\begin{array}{l}
\varepsilon_{1} \\
\varepsilon_{2} \\
\varepsilon_{3} \\
\varepsilon_{4} \\
\varepsilon_{5} \\
\varepsilon_{6}
\end{array}\right]=\left[\begin{array}{cccccc}
s_{11} & s_{12} & s_{12} & 0 & 0 & 0 \\
s_{12} & s_{11} & s_{12} & 0 & 0 & 0 \\
s_{12} & s_{12} & s_{11} & 0 & 0 & 0 \\
0 & 0 & 0 & s_{44} & 0 & 0 \\
0 & 0 & 0 & 0 & s_{44} & 0 \\
0 & 0 & 0 & 0 & 0 & s_{44}
\end{array}\right]\left[\begin{array}{c}
T_{1} \\
T_{2} \\
T_{3} \\
T_{4} \\
T_{5} \\
T_{6}
\end{array}\right](7)
$$

Normalmente, para o silício escolhe-se para a fabricação de sensores a orientação cristalográfica (100), (Akbar, 1991).

Em sensores de pressão, por exemplo, uma máxima sensibilidade à pressão nos diafragmas que usam piezoresistores difundidos tipo $\mathrm{P}$ requer que as bordas sejam alinhadas com a direção $\langle 110\rangle$ e, neste caso, as bordas formam um ângulo $\left(\varnothing=45^{\circ}\right)$ com a direção básica do cristal, cujos eixos, no sistema de coordenadas do diafragma, são girados por um ângulo $(\varnothing)$ com relação aos eixos cristalográficos originais.

A literatura fornece informações sobre muitas das propriedades dos materiais, porém, cabe ao projetista avaliar e determinar aquelas que são importantes para um projeto específico. Neste trabalho pretende-se fazer avaliações e levantar experimentalmente as características relevantes para um projeto de transdutor piezoresistivo de pressão e determinar o fator de sensibilidade dos diferentes materiais usados em consonância com a metodologia proposta.

O diagrama ilustrado na figura 5 mostra como um material com propriedades semicondutora se comporta em termos elétricos, mecânicos e térmicos (Rasia, 1997). A partir desta análise inicia-se o projeto de um transdutor.

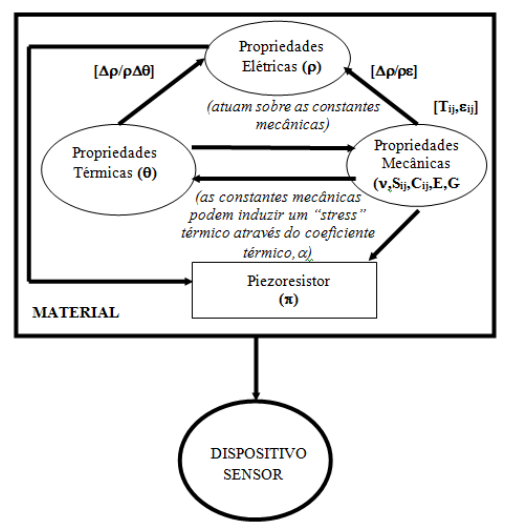

Figura 5. Diagrama dos "efeitos cruzados" em materiais semicondutores

De acordo com o diagrama descrito e considerando que o sinal e a magnitude do efeito piezoresistivo está associada com o tipo de dopante, temperatura, orientação e qualidade cristalográfica (Maisel, 1983) exige-se um alinhamento dos piezoresistores sobre diafragmas com uma grande precisão de posicionamento nas regiões de máximo esforço mecânico (Furlan, 1997) para aperfeiçoar os efeitos resultantes.

Conhecendo-se os momentos de flexão no diafragma analisa-se a distribuição do esforço mecânico sobre o mesmo. As equações que permitem fazer estas análises são dadas por:

$$
\begin{aligned}
& \left(\sigma_{x}\right)_{\text {máx }}=\frac{6 M_{x}}{t^{2}} \\
& \left(\sigma_{y}\right)_{\text {máx }}=\frac{6 M_{y}}{t^{2}}
\end{aligned}
$$

Na Figura 6, é ilustrada esta distribuição de esforços para o eixo x em um diafragma de relação $\frac{a}{b}=1$ cuja pressão aplicada é $P=1,01 \times 10^{5} \mathrm{~N} / \mathrm{m}^{2} \mathrm{e}$ espessura $t=10 \mu m$. As coordenadas servem para posicionamento dos piezoresistores em região de esforço mecânico médio (Rasia, 1997). 


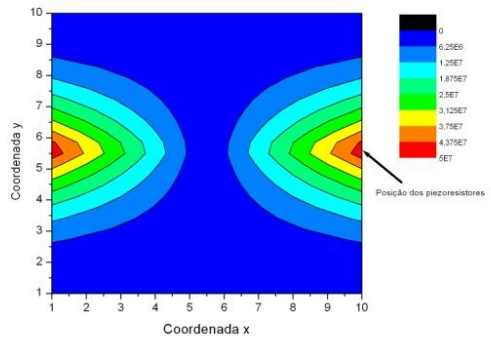

Figura 6. Distribuição de esforços mecânicos nos eixos (xoy)

\section{2 Coeficientes de Rigidez Elásticas ou Coeficiente de Elasticidade}

Os esforços mecânicos em filmes podem ser escritos, de outro modo, em função das deformações mecânicas usando a seguinte equação,

$$
T_{i j}=C_{i j k l} \varepsilon_{i j}
$$

Onde, $C_{i j k l}$, são as constantes de rigidez elásticas. Como não existe uma representação espacial para os tensores de quarta ordem, é conveniente escrever a equação (9) de forma, matricial (Rasia, 1997).

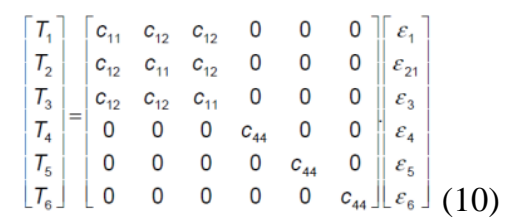

Nessas equações, os índices $i j$ e $k l$ ficaram condensados, assumindo valores entre 1 e 6 , devido à simetria dos tensores. Deste modo, é possível de se fazer uma permutação entre índices sem alterar a propriedade dos mesmos.

\subsection{Propriedades térmicas}

De forma geral, as propriedades térmicas, procuram descrever os efeitos associados à variação da resistência elétrica dos piezoresistores em função da variação da temperatura à qual os mesmos estão submetidos.

Não existe, praticamente, uma propriedade física associada a elementos sensores piezoresistivos integrados que seja completamente independente da temperatura. Portanto; qualquer característica já atribuída aos elementos sensores piezoresistivos é sensível ao efeito da temperatura. Uma medida que venha a ser feita com e em um elemento sensor pode ser afetada pela temperatura e, portanto, sua influência deve ser considerada. Os efeitos da temperatura afetam os sensores e a medida por eles realizados de várias formas (Furlan, 1997, Akbar, 1991).

\section{Descrição dos Modelos Utilizados}

Neste artigo são usados modelos matemáticos de primeira e segunda ordem para analisar resultados experimentais obtidos em trabalhos anteriores (Rasia, 1997).

Um piezoresistor usando um modelo matemático dependente somente dos efeitos da temperatura é dado pela equação:

$$
R(\theta)=R\left(\theta_{\text {ref }}\right) \exp \left[\operatorname{TCR}\left(\theta-\theta_{\text {ref }}\right)\right]
$$

Onde, TCR representa o coeficiente de variação da piezoresistência com a temperatura. De outro modo pode-se usar um modelo mais complexo considerando uma equação de segunda ordem dada por:

$$
R(\theta)=R\left(\theta_{r e f}\right)+\alpha \theta+\beta \theta^{2}
$$

Onde, $\alpha$ e $\beta$ são os coeficientes de variação da resistência com a temperatura, ou seja, os TCRs de primeira e segunda ordem dos piezoresistores.

Entretanto, um piezoresistor mais otimizado é dado pela equação:

$$
\begin{gathered}
R(P, \theta)=R_{r e f}(\theta)\left[1+\pi_{l l}(\theta) T_{l l}(P, \theta)+\right. \\
\left.+\pi_{t}(\theta) T_{t}(P, \theta)+\pi_{x y}(\theta) T_{x y}(P, \theta)\right]
\end{gathered}
$$

Onde, $P$ é a pressão aplicada, $\theta$ é a temperatura, $R_{r e f}$ é a resistência sem esforço mecânico na temperatura de referência $(300 \mathrm{k}), \pi_{x y}$ é o coeficiente piezoresistivo de cisalhamento no plano x-y e $T_{x y}$ é o esforço mecânico de cisalhamento médio (Rasia, 1997).

\section{Resultados das Simulações}

Usando os dados experimentais de um piezoresistor de silício policristalino tipo $\mathrm{P}$ dado por (Rasia, 1997) e o modelo sugerido pela equação (12) obteve-se a curva mostrada na figura 7 . Neste modelo as curvas extrapolam os valores medidos e compreendem uma faixa de temperatura de $-60^{\circ} \mathrm{C}$ a $400^{\circ} \mathrm{C}$, enquanto que, as curvas experimentais estão na faixa de $30^{\circ} \mathrm{C}$ a $270^{\circ} \mathrm{C}$.

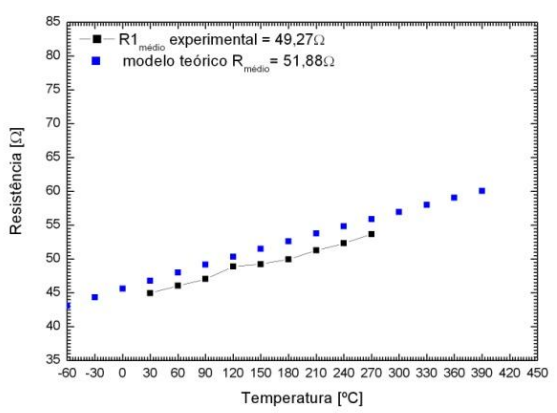

Figura 7. Gráfico da resistência elétrica em função da temperatura para o piezoresistor tipo $\mathrm{P}$. 
Usando o modelo descrito pela equação 13 e considerando os valores mostrados pela figura 3 obteve-se a figura 8 .

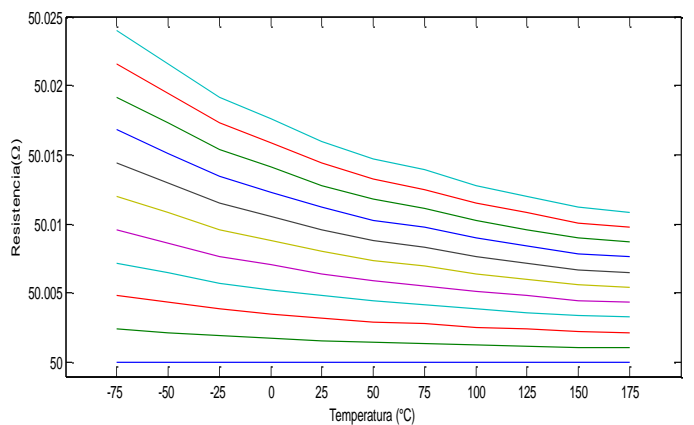

Figura 8. Gráfico da resistência elétrica em função da temperatura

A análise dos resultados simulados e mostrados na figura 8 mostra que, dependendo do nível de dopantes e dos processos de fabricação o piezoresistor tende a assumir um valor médio constante de resistência elétrica para a faixa de temperatura considerada.

\section{Conclusões}

As análises dos resultados simulados mostram que, dependendo do nível de dopantes, faixa de temperatura considerada e dos processos de fabricação o piezoresistor tende a assumir um valor médio constante de resistência elétrica em conformidade com os modelos matemáticos descritos.

\section{Referências Bibliográficas}

RASIA, L. A. Elementos Piezoresistivos para Sensores de Pressão com Tecnologia CMOS. São Paulo, 1997 Dissertação de Mestrado, Escola Politécnica da Universidade de São Paulo.

L.H.van Vlack, Propriedades de Materiais Cerâmicos. Edgard Blücher, São Paulo. 1973.

FRENCH, P.J.; EVANS, G.R. Piezoresistance in polysilicon and its applications to
Strain Gauges. Solid-State Electronics vol.32, N0.1, pp. 1-10, 1989.

DALLY, James W.; RILEY, Wiliam F. Experimental stress analisys McGraw-Hill Inc. Second. Edition, 1978 pp. 205-214.

CIUREANU, P.; MIDDELHOEK, S. Thin films resistive sensors.. Sensors Series. pp. 114; 1992.

BAU, H.H.; de ROOIJ, N.F.; KLOECK, B. Sensors: A comprehensive survey. vol. 7, Mechanical Sensors. Sensors Series. pp. 146-172.

MAISEL, LI e GLANG, R., "Handbook of thin fiIm technology". McGraw-Hillbook Company, 1983.

GNIAZDOWISKI Z; KOWALSKI P., "Practical approach to extraction of piezoresistance coefficient". Institute of Electron Technology, Warsaw (Poland);1998.

L.H.van Vlack, Propriedades de Materiais Cerâmicos. Edgard Blücher, São Paulo. 1973.

Monocristal. Disponivel em http://pt.wikipedia.org/wiki/Monocristal <acessado em 10 de dezembro de $2012>$

Deposição de Filmes Finos. Disponível em http://www.ccs.unicamp.br/cursos/fee107/download/ cap11.pdf <acessado em 19 de março de 2013>

FURLAN, H.; Proposta de implementação de membranas em sensores de pressão a elementos piezoresistivos utilizando a técnica de pósprocessamento. São Paulo, 1997 Dissertação (Mestrado) EPUSP.

CLARK, Samuel K.; WISE, Kensall D. Pressure sensitivity in anisotropically etched thindiaphragm pressure sensors. IEEE Transactions on Electron Devices, vol. ED 26, $\mathrm{N}^{\circ}$. 12, december 1979.

AKBAR, Muhammad. Interface circuit for piezoresistive pressure sensors. Dissertation. Department of Electrical Engineering. University of Michigan, 1991.

KANDA, Y.; A Graphical Representation of the Piezoresistance Coefficients in Silicon. IEEE Transactions on electron devices, vol. Ed-29, no. 1, january 1982. 